\title{
A Note on the Convergence of Asynchronous Greedy Algorithm with Relaxation in a Multiclass Queueing Environment
}

\author{
Wai Ki Ching, Associate Member, IEEE
}

\begin{abstract}
In this letter, we consider the convergence of asynchronous greedy algorithm with relaxation for the Nash equilibrium in a noncooperative multiclass queuing environment. The process of asynchronous greedy algorithm is equivalent to the iteration of Jacobi method in solving a linear system. However, it has been proved that the algorithm converges only for some particular range of queuing parameters. Here we propose the asynchronous greedy algorithm with relaxation, which is in principle equivalent to solving a linear system by Jacobi method with relaxation. We propose also some relaxation parameters such that our algorithm converges very fast.
\end{abstract}

Index Terms-Asynchronous greedy algorithm, Jacobi method, Nash equilibrium.

\section{INTRODUCTION}

I $\mathrm{N}$ THIS letter, we consider the convergence of asynchronous greedy algorithm with relaxation for the Nash equilibrium point in a noncooperative multiclass queuing environment [9]. The problem arises in multiclass telecommunication systems (see, for instance, [2], [3], [9]). The multiclass telecommunication system has $n(n \geq 3)$ independent and noncooperative Poisson streams of packets with arrival rates $\lambda_{1}, \lambda_{2}, \cdots, \lambda_{n}$, respectively. They are served by a single exponential server with service rate $\mu$. To optimize the traffic flow, one may consider the maximization of the throughput or the minimization of the average delay and blocked calls or even a combination of all above. Here each class $i$ of the packets has a performance objective in the maximization of its power, which is defined as the weighted ratio of the throughput over the average delay that the traffic experiences in the queue (see, for instance, [3]). The performance objective of the class $i$ is then given by $P_{i}=r_{i}^{\beta_{i}} /\left(D_{i}\left(r_{1}, r_{2}, \cdots, r_{n}\right)\right)$ where the parameters are defined as follows:

1) $r_{i}$ is the resulting throughput of the class $i$;

2) $D_{i}\left(r_{1}, r_{2}, \cdots, r_{n}\right)$ is the average delay;

3) $\beta_{i}$ is the positive weighting factor.

The Nash equilibrium point is defined as the point where no user would unilaterally deviate and any deviation would result

Manuscript received December 2, 1997. The associate editor coordinating the review of this letter and approving it for publication was Prof. C. Dougligeris. This work was supported by the Hong Kong Croucher Foundation and RGC earmarked Grant CUHK 4125/97E.

The author is with the Department of Mathematics, Hong Kong University of Science and Technology, Clear Water Bay, Hong Kong, and also with the Department of Systems Engineering and Engineering Management, Chinese University of Hong Kong, Shatin, Hong Kong (e-mail: wkching@se.cuhk.edu.hk).

Publisher Item Identifier S 1089-7798(99)01255-7. in worsening of its performance objective, see [9]. For more detail about the Nash equilibrium, see [5]-[7].

Under the assumption that no cooperation is allowed and the users are symmetric in the information they acquire, then each user would try to reach a point so that its performance is optimal in the sense that any unilateral derivation from that point would render it worse. Then this point is called the Nash equilibrium point. It turns out that the Nash equilibrium point is the solution of the following linear equation: $A_{n} \mathrm{x}=\mathrm{b}$, where

$$
\begin{aligned}
A_{n} & =\left[\begin{array}{cccc}
1 & \frac{\beta_{1}}{\left(\beta_{1}+1\right)} & \cdots & \frac{\beta_{1}}{\left(\beta_{1}+1\right)} \\
\frac{\beta_{2}}{\left(\beta_{2}+1\right)} & 1 & \cdots & \frac{\beta_{2}}{\left(\beta_{2}+1\right)} \\
\vdots & \vdots & \ldots & \vdots \\
\frac{\beta_{n}}{\left(\beta_{n}+1\right)} & \cdots & \frac{\beta_{n}}{\left(\beta_{n}+1\right)} & 1
\end{array}\right] \\
\mathbf{b} & =\left[\begin{array}{c}
\frac{\beta_{1} \mu}{\left(\beta_{1}+1\right)} \\
\frac{\beta_{2} \mu}{\left(\beta_{2}+1\right)} \\
\vdots \\
\frac{\beta_{n} \mu}{\left(\beta_{n}+1\right)}
\end{array}\right] .
\end{aligned}
$$

Here each weighting factor $\beta_{i}$ is positive. It has been shown in [2] the Nash equilibrium point is given by

$$
x_{i}=\beta_{i} \mu /\left(1+\sum_{k=1}^{n} \beta_{k}\right), \quad \text { for } i=1,2, \cdots, n \text {. }
$$

We are interested in obtaining fast and convergent algorithm for the communication system to attain this equilibrium point.

The Nash equilibrium point can be attained by using the synchronous greedy algorithm, see [9]. The algorithm is equivalent to the procedure of solving the linear system (1) by the Gauss-Seidel method in principle. In real network environment, to implement the synchronous greedy algorithm, each user $i$ needs to know his cost function and the service rate and the throughput of other users. Thus in each time, each user has to compute its update and wait until the other users have finished their updates. Therefore, seeking an asynchronous algorithm is natural. Here, the asynchronous greedy algorithm is considered, which is equivalent to the procedures of solving the linear system (1) by the Jacobi method. Unfortunately, it has been proved that the asynchronous greedy algorithm 
converges if and only if (see [9])

$$
\sum_{i=1}^{n} \frac{1}{1+2 \beta_{i}}>n-2, \quad n \geq 2 .
$$

To cope with this limitation, we consider the asynchronous greedy algorithm with relaxation, which is equivalent to solving the linear system (1) by the Jacobi (JOR) method with relaxation. By choosing suitable relaxation, we prove that our JOR algorithm converges. The relaxed Jacobi iteration matrix is then given by

$$
J_{n}=I_{n}-\omega A_{n}
$$

where $I_{n}$ is the $n \times n$ identity matrix and $0<\omega \leq 1$. We note that the relaxed asynchronous greedy algorithm converges if and only if the JOR method converges. The sufficient condition for the JOR to converge is that the spectral radius $\rho\left(J_{n}\right)$ of $J_{n}$ is strictly less than 1 , see [1] for instance.

The remainder of the paper is organized as follows. In Section II, we give some upper bounds for the spectral radius of the matrix $J_{n}$. We give the optimal relaxation parameter which minimizes the bounds and prove that the JOR method converges under the relaxation parameter.

\section{SPECTRAL RADIUS BOUNDS AND OPTIMAL RELAXATION PARAMETER}

In this section, we give some upper bounds for the spectral radius of the matrix $J_{n}$ [cf. (2)]. We give the optimal relaxation parameter which minimizes the spectral bounds and prove that the JOR method converges under the proposed relaxation parameter. For simplicity and ease of presentation, we let

$$
q_{i}=\frac{\beta_{i}}{\beta_{i}+1}>0, \quad \text { for } i=1,2, \cdots, n
$$

and, therefore, we have

$$
J_{n}=\left[\begin{array}{ccccc}
1-\omega & -\omega q_{1} & \cdots & \cdots & -\omega q_{1} \\
-\omega q_{2} & 1-\omega & -\omega q_{2} & \cdots & -\omega q_{2} \\
\vdots & \vdots & \ddots & \vdots & \vdots \\
\vdots & \vdots & \vdots & \ddots & \vdots \\
-\omega q_{n} & \cdots & \cdots & -\omega q_{n} & 1-\omega
\end{array}\right] .
$$

We first give a lemma concerning the eigenvalues of the matrix $J_{n}$.

Lemma 1: The matrix $J_{n}$ is similar to a symmetric matrix and hence all the eigenvalues of $J_{n}$ are real.

Proof: Let

$$
D_{n}=\operatorname{diag}\left(\sqrt{q_{1}}, \sqrt{q_{2}}, \cdots, \sqrt{q_{n}}\right)
$$

then $S_{n} \equiv D_{n}^{-1} J_{n} D_{n}$ is a symmetric matrix and

$$
\begin{aligned}
& S_{n}=\left[\begin{array}{ccc}
1-\omega\left(1-q_{1}\right) & & 0 \\
0 & \ddots & 1-\omega\left(1-q_{n}\right)
\end{array}\right] \\
& -\omega\left[\begin{array}{c}
\sqrt{q_{1}} \\
\vdots \\
\sqrt{q_{n}}
\end{array}\right]\left[\begin{array}{lll}
\sqrt{q_{1}} & \cdots & \sqrt{q_{n}}
\end{array}\right] .
\end{aligned}
$$

Hence Lemma 1 is proved.

The JOR method converges if the spectral radius of $J_{n}$ is strictly less than one [1]. We remark that the smaller the value of $\rho\left(J_{n}\right)$, the faster the convergence rate of the JOR

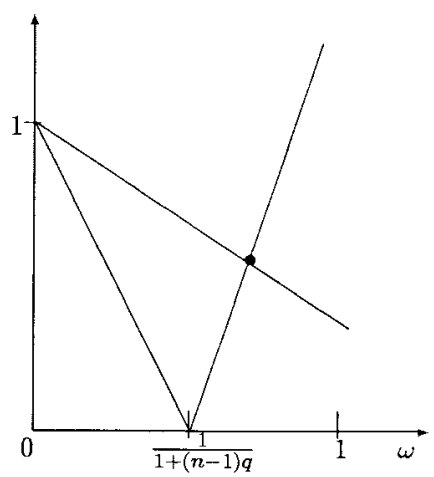

Fig. 1. Graph of $f(\omega)$ and $g(\omega)$.

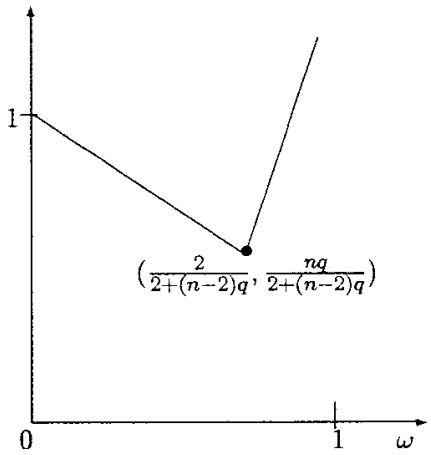

Fig. 2. Graph of $\operatorname{Max}\{f(\omega), g(\omega)\}$.

method. We first consider the case when all the $\beta_{i}$ are equal. The following theorem gives the optimal relaxation parameter $\omega^{*}$ which minimizes $\rho\left(J_{n}\right)$ for this case.

Theorem 1: If $q_{1}=q_{2}=\cdots=q_{n}=q$, then the optimal relaxation parameter $\omega^{*}$ which minimizes $\rho\left(J_{n}\right)$ is given by

$$
\frac{2}{2+(n-2) q}
$$

and the optimal spectral radius is

$$
\lambda^{*}=\frac{n q}{2+(n-2) q} .
$$

Proof: From (5), we see that the eigenvalues are given by

$$
1-\omega(1-q) \text { and } 1-\omega(1+n q-q) \text {. }
$$

In the following we give the graphs of $f(\omega)=|1-\omega(1-q)|$, $g(\omega)=|1-\omega(1+n q-q)|$, and $\operatorname{Max}\{f(\omega), g(\omega)\}$.

Therefore, we have

$$
\lambda^{*}=\min _{0<\omega \leq 1}\{\max \{|1-\omega(1-q)|,|1-\omega(1+n q-q)|\}\} \text {. }
$$

By straightforward computation or the graphs above, we see that the optimal relaxation parameter which minimizes $\rho\left(J_{n}\right)$ is

$$
\omega^{*}=\frac{2}{2+(n-1) q}
$$

and the optimal spectral radius is given by

$$
\lambda^{*}=\frac{n q}{2+(n-2) q} \text {. }
$$

Notice that $0<q<1$ and therefore $\lambda^{*}<1$. Thus we have the following corollary. 
Corollary 1: If $q_{1}=q_{2}=\cdots=q_{n}$ and the relaxation parameter is chosen as $\omega^{*}$ in Theorem 1, then the JOR method converges independent of the initial states.

Let us consider the general situation that all the weighting factors $\beta_{i}$ are not necessary identical. We first define the following notations:

$$
\begin{aligned}
q_{\max } & =\max \left\{q_{1}, q_{2}, \cdots, q_{n}\right\} \\
q_{\min } & =\min \left\{q_{1}, q_{2}, \cdots, q_{n}\right\},
\end{aligned}
$$

(cf. 3). Before we give an upper bound and a lower bound for the eigenvalues of $J_{n}$, we need the following lemma.

Lemma 2 (Weyl's Theorem): Let $A$ and $B$ be two $n \times n$ real symmetric matrices and let the eigenvalues $\lambda_{i}(A), \lambda_{i}(B)$ and $\lambda_{i}(A+B)$ be arranged in increasing order. Then for each $k=1,2, \cdots, n$ we have

$$
\lambda_{k}(A)+\lambda_{1}(B) \leq \lambda_{k}(A+B) \leq \lambda_{k}(A)+\lambda_{n}(B) .
$$

Proof: See [4].

Theorem 2: All the eigenvalues of $J_{n}$ lie in the interval

$$
\left[1-\omega\left(1-q_{\min }+\sum_{i=1}^{n} \sqrt{q_{i}}\right), 1-\omega\left(1-q_{\max }\right)\right] .
$$

Proof: Since $S_{n}$ is similar to $J_{n}$, the two matrices $J_{n}$ and $S_{n}$ have same spectrum (eigenvalues). Notice that

$$
\begin{aligned}
S_{n}= & {\left[\begin{array}{ccc}
1-\omega\left(1-q_{1}\right) & & 0 \\
& \ddots & \\
0 & & 1-\omega\left(1-q_{n}\right)
\end{array}\right]-\omega\left[\begin{array}{c}
\sqrt{q_{1}} \\
\vdots \\
\sqrt{q_{n}}
\end{array}\right] } \\
& \cdot\left[\begin{array}{lll}
\sqrt{q_{1}} & \cdots & \sqrt{q_{n}}
\end{array}\right]
\end{aligned}
$$

where $E_{n}$ is a diagonal matrix and $F_{n}$ is rank one matrix. Thus both of them are symmetric matrices. Clearly the eigenvalues of $E_{n}$ are

$$
1-\omega+\omega q_{i}, \quad i=1,2, \cdots, n .
$$

Thus the maximum and minimum eigenvalues of $E_{n}$ are $\left(1-\omega+\omega q_{\max }\right)$ and $\left(1-\omega+\omega q_{\min }\right)$, respectively. Moreover, the eigenvalues of $F_{n}$ are 0 and $\sum_{i=1}^{n} \sqrt{q_{i}}$.

Hence the maximum and minimum eigenvalues of $F_{n}$ are $\sum_{i=1}^{n} \sqrt{q_{i}}$ and 0 , respectively. We apply the Weyl's Theorem (Lemma 2) to $S_{n}$. Let $A=E_{n}$ and $B=-\omega F_{n}$. Then by letting $k=n$ and $k=1$ we get the upper and lower bounds of the eigenvalues of $S_{n}$, respectively. Thus we have all the eigenvalues of $J_{n}$ lie in the interval

$$
\left[1-\omega\left(1-q_{\min }+\sum_{i=1}^{n} \sqrt{q_{i}}\right), 1-\omega\left(1-q_{\max }\right)\right] .
$$

Hence our claim is proved.

Thus we have

$$
\begin{array}{r}
\rho\left(J_{n}\right) \leq R(\omega)=\max \left\{\left|1-\omega\left(1-q_{\min }+\sum_{i=1}^{n} \sqrt{q_{i}}\right)\right|,\right. \\
\left.\left|1-\omega\left(1-q_{\max }\right)\right|\right\} .
\end{array}
$$

We seek for relaxation parameter which minimizes the upper bound $R(\omega)$ for $\rho\left(J_{n}\right)$. By considering the graph of $R(\omega)$, one can have the following corollary.

Corollary 2: The relaxation parameter which minimizes $R(\omega)$ is given by

$$
\begin{aligned}
\omega^{* *} & =\frac{2}{2-q_{\max }-q_{\min }+\sum_{i=1}^{n} \sqrt{q_{i}}} \\
r & =R\left(\omega^{* *}\right)=1-\frac{2\left(1-q_{\max }\right)}{2-q_{\max }-q_{\min }+\sum_{i=1}^{n} \sqrt{q_{i}}}<1 .
\end{aligned}
$$

We see that when the relaxation parameter is given by $\omega^{* *}$, all the eigenvalues of $J_{n}$ lie in the interval $[-r, r]$. Hence $\rho\left(J_{n}\right) \leq r<1$ and the JOR method converges. In general, even if we have no information of the parameters $q_{\max }$ and $q_{\min }$, we may choose the following relaxation parameter $\omega^{+}=$ $2 /(n+1)$. From Theorem 2, we have the following corollary.

Corollary 3: If the relaxation parameter is chosen to be $\omega^{+}=2 /(n+1)$, then all the eigenvalues of $J_{n}$ lie in the interval

$\left[1-\frac{2\left(1-q_{\min }+\sum_{i=1}^{n} \sqrt{q_{i}}\right)}{n+1}, 1-\frac{2\left(1-q_{\max }\right)}{n+1}\right] \subset(-1,1)$.

Hence the JOR method converges under the relaxation parameter $\omega^{+}$.

\section{ACKNOWLEDGMENT}

The author would like to thank Prof. C. Douligeris and Prof. R. H. Chan for their helpful suggestions.

\section{REFERENCES}

[1] O. Axelsson, Iterative Solution Methods. Cambridge, U.K.: Cambridge Univ. Press, 1996

[2] C. Douligeris and R. Mazumdar, "A game theoretic perspective to flow control in telecommunication networks," J. Franklin Inst., vol. 329, no. 2, pp. 383-402, 1992.

[3] A. Giessler, J. Hanle, A. Konig, and E. Pade, "Free buffer allocation: An investigation by simulation," Computer Networks, vol. 1, no. 3, pp. 199-204, 1978

[4] R. Horn and C. Johnson, Matrix Analysis. Sydney, Australia: Cambridge Univ. Press, 1991, pp. 184-185.

[5] L. Kumar, C. Douligeris, and G. Develekos, "Implementation and performance analysis of a decentralized multiclass flow control algorithm using pareto optimal criterion," Computer Commun., vol. 17, no. 8, pp. 600-610, 1994.

[6] P. Morris, Introduction to Game Theory. New York: Springer-Verlag, 1994, pp. 115-147.

[7] J. Nash, "Noncooperative games," Ann, Math., vol. 21, pp. 128-140, 1953.

[8] R. Varga, Matrix Iterative Analysis. Englewood Cliffs, NJ: PrenticeHall, 1963.

[9] Z. Zhang and C. Douligeris, "Convergence of synchronous and asynchronous greedy algorithm in a multiclass telecommunications environment," IEEE Tran. Commun., vol. 40, pp. 1277-1281, 1992. 